\title{
Neun gute Gründe, Unterschriften für die Initiative zu sammeln
}

Initiativkomitee Transparenz in der Krankenversicherung

\section{Zwei Versicherungen - zwei unterschiedliche Ansätze}

Die obligatorische Krankenversicherung nach KVG ist eine Sozialversicherung und als solche der Solidarität verpflichtet. Sie wird hauptsächlich über einen obligatorischen Beitrag, eine Kopfprämie mit Steuercharakter, finanziert. Der Leistungskatalog, auf den sie sich bezieht, ist für alle gleich.

Die Zusatzversicherungen nach VVG sind hingegen freiwillig. Sie gestatten es dem Versicherer, einen Gewinn zu erwirtschaften. Bei den Zusatzversicherungen dürfen die Krankenkassen Bewerber ablehnen oder Gesundheitsvorbehalte anbringen.

Nach derzeitiger Regelung sind die Krankenkassen berechtigt, diese beiden Versicherungsformen zugleich zu betreiben.

\section{Unzureichende wirtschaftliche Transparenz und ungenügende Überwachung}

Die Grundversicherung muss völlig transparent und unabhängig von jeder anderen Geschäftstätigkeit geführt werden. Diese Transparenz ist für einen gesunden und wirtschaftlichen Umgang mit den Prämiengeldern unerlässlich. Doch die Aufsicht des Bundesamtes für Gesundheit (BAG) genügt bei dem heutigen System nicht für eine wirksame und zufriedenstellende Überwachung dieser Gelder. Im BAG sind lediglich 15 Personen mit der Überwachung der 81 Krankenkassen betraut, die insgesamt 20 Milliarden Prämienfranken für die Grundversicherung kassieren (Zahlen des BAG für 2009).

Trotz der seit Jahren angebrachten Beanstandungen werden die Prämien weiterhin nach intransparenten Methoden und nicht im Verhältnis zu den tatsächlichen Kosten festgelegt. Die Affäre um die Reservenüberschüsse, die aus geschäftsstrategischen Gründen in bestimmten Kantonen gebildet werden und in andere Kantone abfliessen, führt die unbillige Geschäftsführung der Krankenversicherer klar vor Augen.

\section{Pseudo-Synergien und tatsächliche Intransparenz}

Wären private Anbieter wie die heutigen Krankenkassen tatsächlich in der Lage, die obligatorische Grundversicherung wirksam zu betreiben, müssten sie
Eidgenössische Volksinitiative «Für Transparenz in der Krankenversicherung"

Die Ärzte der drei Sprachregionen der Schweiz lancieren mit Unterstützung der Verbindung der Schweizer Ärztinnen und Ärzte FMH eine eidgenössische Volksinitiative. Ziel ist die vollständige Trennung zwischen der obligatorischen Krankenversicherung als Sozialversicherung einerseits und den Zusatzversicherungen andererseits. So können die Krankenkassen nicht mehr diese beiden, vom Gesetz her unterschiedlichen Versicherungstypen zugleich anbieten. Sie werden sich für die eine oder die andere Geschäftstätigkeit entscheiden müssen.

eigentlich ihre Geschäftsführung völlig transparent gestalten. Die konsolidierten Jahresrechnungen der Krankenversicherungen taugen jedoch nicht als Beleg für die Übereinstimmung ihrer Abrechnungsmethoden mit dem KVG, denn die Revisionsgesellschaften sind nicht verpflichtet, diese Übereinstimmung zu verifizieren. Die Vermischung, die sich ergibt, wenn die Krankenkassen Grundversicherung und Zusatzversicherungen mit demselben Personal und derselben Infrastruktur (Gebäude, Informatik) betreiben, gestattet jedenfalls keine echte Transparenz. Die Intransparenz, die sich aus dem Miteinander zweier eigentlich gegenläufiger Versicherungsformen ergibt, hat das System in seinem Kern lahmgelegt. Im Zusammenspiel mit einer mangelhaften Überwachung verleiten solche «Synergien» die Versicherer nicht nur zur Risikoselektion, sondern sie drängen sie geradezu, Aufwand, Leistungen und Mittel auf die Grundversicherung zu übertragen und so zur künstlichen Erhöhung der Prämien beizutragen. Die Geldflüsse innerhalb der grossen Krankenkassen sind derart undurchsichtig, dass sie sich mittlerweile jeder politischen Kontrolle entziehen. Diese Intransparenz schlägt sich sogar in der Rechtsstruktur nieder, die sich gewisse grosse Versicherungsgruppen in der fast unverhohlenen Absicht gegeben haben, sich der Aufsicht des BAG zu entziehen.

Obschon sie sich auf ihre Gutgläubigkeit berufen, lehnen die Versicherer jeden Wunsch nach mehr 
Durchblick ab unter dem Vorwand eines «Geschäftsgeheimnisses», das sie sich auf die Verwaltung einer obligatorischen Sozialabgabe anzuwenden berechtigt meinen.

\section{Keine Verknüpfung mehr zwischen dem Abschluss einer Zusatzversicherung und der Grundversicherung}

Der Leistungskatalog der Grundversicherung, das Verzeichnis der vergüteten medizinischen Leistun- dass der neue, vermutlich ab 2012 gültige Risikoausgleich solchen Machenschaften ein Ende setzen wird.

Tatsächlich profitieren die Krankenversicherer also nur selber von den sogenannten Synergien, die sie als wichtigsten Grund für die Beibehaltung des Status quo anführen, während die Versicherten lediglich Nachteile davontragen. Die Grundversicherung wird damit zum Lockvogel für den Verkauf von $\mathrm{Zu}$ satzversicherungen degradiert. So erklären sich die Kosten von schätzungsweise fast 100 Millionen Fran-

\section{«Die Versicherten müssen sich frei fühlen, ihre Verträge beim besten Grundversicherer abzuschliessen, ohne sich durch die Zusatzversicherung gebunden zu fühlen.»}

gen, ist für alle gleich. Der Zusatzversicherer verfügt somit über alle Angaben, die er benötigt, um zu erkennen, welche Kosten ihm und welche der Grundversicherung zufallen. Es bringt daher nichts, beide Verträge bei demselben Versicherer zu halten. Eine qualitätsbewusste Zusatzversicherung im Sinne des Versicherten ist eine, die diese Unterscheidung klar vornimmt.

Die Versicherten müssen sich frei fühlen, ihre Verträge beim besten Grundversicherer abzuschliessen, ohne sich durch die Zusatzversicherung gebunden zu fühlen. Es ist nicht annehmbar, dass sie, wenn sie völlig legal in den Genuss einer günstigeren Prämie kommen möchten, von einem Wechsel der Grundversicherung abgehalten werden, weil ihnen mit einer Erhöhung der Prämie für die Zusatzversicherung oder dem Entzug entsprechender Rabatte gedroht wird. Diese Zwangsbindung stellt eine gravierende Wettbewerbsverzerrung zu Ungunsten der Versicherten dar.

\section{Risikoselektion, Blendwerk und Schikanen}

Während bei der Grundversicherung Aufnahmezwang besteht und auch keine Vorbehalte gemacht werden dürfen, ist für die Zusatzversicherung die Risikoselektion anhand von Fragebogen und Gesundheitstests durchaus gestattet. Zahlreiche Erfahrungsberichte zeigen, dass einmal als «schlechte Risiken» identifizierte Anwärter, die voraussichtlich auch ohne eigenes Verschulden teuer zu kosten kommen, keine Antwort auf ihren Aufnahmeantrag in die Grundversicherung erhalten und dass niemand das Telefon abnimmt, wenn sie anrufen. In der Absicht, die «guten Risiken» herauszupicken, nutzen einige Versicherungsgesellschaften die Gelegenheit eines Übertritts, um ihren zukünftigen Grundversicherten über eine unbedeutende Alibi-Zusatzversicherung illegal Angaben zu ihrer Gesundheit zu entlocken. Die meisten Experten halten es für hochgradig unwahrscheinlich, ken, die allein für Werbung und Kundenakquisition beim Verkauf eines Produktes anfallen, das doch bei allen Versicherern identisch ist.

\section{Mangelhafter Datenschutz}

Die Informationen, die der Versicherer im Zusammenhang mit den Zusatzversicherungen erlangt, dürften nicht dazu dienen, jemanden von einem Wechsel der Grundversicherung abzuhalten. Und doch sind, wie viele Zeugen unter anderem in Medienberichten bestätigen, diese eigentlich streng geschützten Daten innerhalb einer Versicherung weitgehend verfügbar, denn die beiden Versicherungssysteme werden oft demselben Personal anvertraut. Ab 2012, wenn das neue Spital-Tarifsystem (DRG) in Kraft tritt, werden die Versicherer noch vermehrt Zugang zu sensiblen Gesundheitsdaten erhalten, und das Risiko einer Verwendung gegen die Interessen der Versicherten wird erheblich zunehmen. Somit kann die Doppelfunktion der Versicherungsgesellschaften als wahres Damoklesschwert den Ausschluss des Versicherten von einer Zusatzversicherung oder die Verweigerung von Leistungen bewirken.

\section{Die Versicherten kennen die Unterschiede zwischen den beiden Versicherungsarten nicht}

Die gesetzliche Grundlage für die obligatorische Krankenversicherung ist das Krankenversicherungsgesetz (KVG), für die Zusatzversicherungen hingegen das Versicherungsvertragsgesetz (VVG). Wenn jedoch ein Versicherungsvertrag eine kombinierte Deckung vorsieht, können Laien nur schwerlich erkennen, welche Bestimmungen sich worauf beziehen. Ein Verbot von Mischverträgen könnte auch hier Abhilfe schaffen und verhindern, dass diese Verwirrung dazu genutzt wird, von den Versicherten mehr oder weniger legal persönliche Daten einzuholen. 


\section{Es geht um die Zukunft der Kranken- versicherung}

Infolge des heutigen Finanzierungsmodus der obligatorischen Krankenkasse sind immer mehr Versicherte, darunter insbesondere Familien, kaum mehr in der Lage, ihre Prämien zu zahlen, und alle Szenarien deuten auf eine weitere Verteuerung des Gesundheitswesens hin. Eine wirksame Umsetzung der Reformen, die im Gange sind, um diesen Kosten Einhalt zu gebieten, ist nur auf der Grundlage eines gesunden Finanzierungssystems möglich. Eine transparente

\section{Im Interesse der Versicherten}

Durch die Trennung von Zusatzversicherungen und Grundversicherung wird den Verzerrungen, die sich aus deren Vermischung ergeben, Einhalt geboten, und die Grundversicherung wird wieder ihrer wahren Bestimmung als Sozialversicherung auf Gegenseitigkeit zugeführt. So kann die politische Debatte wieder mit der für die Fortführung der Reformen erforderlichen Gelassenheit geführt werden. Doch in erster Linie liegt diese Trennung im Interesse der Versicherten selbst, denn sie schafft eine klare Regelung, die auf

\section{Durch die Trennung von Zusatzversicherungen und Grundversicherung wird den Verzerrungen, die sich aus deren Vermischung ergeben, Einhalt geboten.}

Krankenversicherung ist daher unabdingbar für die Wiederherstellung des Vertrauens und der Glaubwürdigkeit, ohne die keine der Reformen durchzuziehen ist, deren unser Gesundheitswesen bedarf, sollen die Prämien massvoll bleiben.

Die politischen Debatten haben ein wahres Netzwerk von politischen Seilschaften ans Licht gebracht, das die Versicherer in Bern aufgebaut haben, um ihre Kontrolle über das Gesundheitswesen zu festigen und jegliche Reform im Sinne einer vermehrten Transparenz abzuwürgen. Diese mächtige, prämienfinanzierte Lobby war verantwortlich für zahlreiche Blockierungsmanöver und die folgliche Verewigung unhaltbarer Zustände für die Versicherten. politischem Wege mit allen Verzögerungen und Halbheiten nicht zu erreichen war. Sie macht Schluss mit den schädlichen Anreizen, die bewirken, dass zwischen den Interessen der Krankenversicherer und jenen ihrer Versicherten ein grundsätzlicher Widerspruch besteht.

Das Parlament hat sich bereits mit drei Vorstössen zugunsten einer verbesserten Transparenz befasst. Alle wurden schliesslich zurückgezogen oder abgelehnt, ohne dass die nötigen Massnahmen ergriffen worden wären, um transparente Abläufe in der obligatorischen Krankenversicherung zu gewährleisten. Da noch jedes Projekt in diesem Sinne an der parlamentarischen Lobby gescheitert ist, muss sich das Volk nun selbst Gehör verschaffen.

\section{Sammeln Sie deshalb Unterschriften für die Initiative «Für Transparenz in der Kranken- versicherung»! \\ Sie können die Unterschriftenliste in diesem Heft verwenden oder weitere Unterschriften- listen von der Internetseite: http://initiative- transparenz.ch herunterladen und ausdrucken.}

Sichtbar getragene Selbstklebe-Etiketten erleichtern den Einstieg in das Gespräch!
UNTERSCHREIBEN! initiative-transparenz.ch

SIGNEZ!

initiative-transparence.ch

FIRMARE!

iniziativa-trasparenza.ch 